WIS-93/98/OCT-PH

July 15, 2021

\title{
Supersymmetry and Light Quark Masses in a Realistic Superstring Model
}

\author{
EDI HaLyo ${ }^{\dagger}$ \\ Department of Physics, Weizmann Institute of Science \\ Rehovot 76100, Israel
}

\begin{abstract}
We examine the light quark masses in a standard-like superstring model in the four dimensional free fermionic formulation. We find that the supersymmetry constraints in the observable and hidden sectors eliminate all large contributions to $m_{u}$ and $m_{d}$ and force them to be much smaller than the other quark masses. The requirement for an acceptable Higgs doublet spectrum results in $m_{u}<<m_{d}$. In these models a realistic $m_{d}$ can always be obtained whereas $m_{u}$ is at most $10^{-5} \mathrm{MeV}$. For particular choices of flat directions or vacua $m_{u}$ can be as small as $10^{-7} \mathrm{MeV}$ but cannot vanish.
\end{abstract}

\footnotetext{
$\dagger$ e-mail address: jphalyo@weizmann.bitnet
} 


\section{Introduction}

One of the many puzzling features of the quark spectrum is the smallness of the (current) up and down quark masses. These are not only suppressed by a factor of $\sim 10^{-4}-10^{-5}$ with respect to the weak scale but are also much smaller than the other quark masses. According to 't Hooft's naturalness criterion [1], the smallness of $m_{u}$ and $m_{d}$ must follow from symmetries which are broken only by a very small amount in order to result in such small $m_{u}$ and $m_{d}$. In the limit of exact symmetry one would expect to have vanishing $m_{u}$ and $m_{d}$. Thus, a small or vanishing $m_{u}$ is only natural if it is a result of some (possibly discrete) symmetry. Another aspect of a small or vanishing $m_{u}$ is that $m_{u}=0$ is a possible solution to the strong CP problem [2]. By now, it is well known that a vanishing $m_{u}$ is not in conflict with current algebra results [3].

Any extension of the standard model which tries to explain the origin of fermion masses must explain or at least accomodate the light fermion masses. Foremost among these are superstring theories [4]. Certainly, if superstring theories are "the theories of everything", they should explain the smallness of $m_{u}$ and $m_{d}$ in addition to the rest of the quark spectrum. It is therefore important to examine light quark masses in realistic superstring models. The purpose of this work is to see whether current up and down quark masses can be obtained in standard- like superstring models. Moreover, we would like to know if a vanishing $m_{u}$ is possible. We also hope to gain an understanding of the symmetries which cause the light quark masses to be much smaller than those of other quarks.

The standard-like superstring model that we consider has the following properties $[5,6]$ :

1. $N=1$ space-time supersymmetry (SUSY).

2. A $S U(3)_{C} \times S U(2)_{L} \times U(1)^{n} \times$ hidden gauge group.

3. Three generations of chiral fermions and their superpartners, with the correct quantum numbers under $S U(3)_{C} \times S U(2)_{L} \times U(1)_{Y}$. 
4. Higgs doublets that can produce realistic electro-weak symmetry breaking.

5. Anomaly cancellation, apart from a single "anomalous" $\mathrm{U}(1)$ which is canceled by application of the Dine-Seiberg-Witten (DSW) mechanism [7].

As noted above, in standard-like superstring models there are no gauge and gravitational anomalies apart from a single "anomalous $U(1)$ " symmetry. This anomalous $U(1)_{A}$ generates a Fayet-Iliopoulos term that breaks SUSY at the Planck scale [7]. SUSY is restored and $U(1)_{A}$ is broken by giving VEVs to a set of standard model singlets in the massless string spectrum along the flat $\mathrm{F}$ and D directions [8]. Thus, the $S O(10)$ singlet fields in the non-renormalizable terms obtain non-vanishing VEVs by the application of the DSW mechanism. In addition, scalars which are in the vector representations of unbroken, nonAbelian, hidden sector gauge groups condense and obtain VEVs at the condensation scale, $\Lambda_{H}$. Then, the order $N(=m+n)$ non-renormalizable terms, of the form $\operatorname{cffh}\left(\Phi^{m} V^{n} / M\right)^{N-3}$ (for $n=0,2$ ), become effective Yukawa terms, where $f, h, \Phi, V$ denote fermions, scalar doublets, scalar singlets and hidden sector states, respectively. $M$ is a Planck scale mass to be defined later. The effective Yukawa couplings are given by $\lambda=c\left(\left\langle\Phi^{m} V^{n}\right\rangle / M\right)^{N-3}$ where the calculable coefficients $c$ are of order one [9]. In this manner quark mass terms, as well as quark mixing terms, can be obtained. Realistic quark mixing and masses for the two heavy generations have been obtained for a suitable choice of scalar VEVs [10].

In Ref. (11) the quark mass hierarchy for the heaviest two generations was obtained by giving mass from the cubic superpotential only to the top quark. The other quarks except the light ones (i.e. $u$ and $d$ ) get their masses from $N=5$ non-renormalizable terms and hence they are suppressed relative to the top mass by a factor of $\left\langle\Phi^{2}\right\rangle / M^{2} \sim 10^{-2}-10^{-3}$. It was also noticed that light quark masses cannot arise only from the observable sector VEVs, to any order in $N$, due to SUSY constraints in the observable sector.

In this work we examine the contributions to light quark masses that arise from hidden sector VEVs in addition to those from the observable sector. We find 
that SUSY constraints in the observable and hidden sectors (with the requirement of realistic heavy quark masses) eliminate the potentially large contributions to up and down masses. In fact, SUSY constrains $m_{u}$ and $m_{d}$ to be around the $M e V$ scale. This can be explained as a result of an effective $Z_{4}$ symmetry arising from the SUSY constraints. There are two possible scenarios in which either $\bar{h}_{1}$ or $\bar{h}_{2}$ is the light Higgs doublet that couples to the up-like quarks. In both cases the doublet that couples to down-like quarks is $h_{45}$. We find that a realistic $m_{d} \sim$ $\mathrm{MeV}$ can be obtained in both cases from the off-diagonal terms in the down quark mass matrix. On the other hand, we find that $m_{u}$ is at most $\sim 10^{-5} \mathrm{MeV}$ which is six orders of magnitude smaller than its value obtained from current algebra i.e. $\sim 5 \mathrm{MeV}$ but not small enough to solve the strong $\mathrm{CP}$ problem naturally. If we take $\left\langle\bar{\Phi}_{3}^{+}\right\rangle=0$, then the only contribution to $m_{u}$ comes from $N=8$ terms which contain hidden sector condensates and give $m_{u} \sim 10^{-6} \mathrm{MeV}$. We also find that $m_{u}$ vanishes up to $N=8$ for a flat direction with $\left\langle\Phi_{3}^{ \pm}\right\rangle=\left\langle\bar{\Phi}_{3}^{ \pm}\right\rangle=0$. There are always, non-zero, order $N>8$ terms which contribute to $m_{u}$ and therefore $m_{u}$ cannot vanish to all orders in $N . N=9$ terms give a lower bound of $m_{u} \sim 10^{-7} \mathrm{MeV}$. $m_{u}<<m_{d}$ in this model since the only $M e V$ scale contribution to $m_{u}$ vanishes due to the constraints from an acceptable Higgs doublet spectrum as we show below.

The paper is organized as follows. In Section 2, we review the superstring model. In Section 3, we find the SUSY constraints on VEVs of the hidden sector states in addition to those of the observable sector states. In Section 4, we examine the Higgs doublet mass matrix and find the constraints for a realistic Higgs doublet spectrum. In Section 5, we obtain the up and down quark mass matrices and examine the contributions to the light quark masses in detail. We also consider all possible kinds of non-renormalizable terms which contribute to $m_{u}$ and $m_{d}$. In Section 6, we present a discussion and our conclusions.

\section{The superstring model}

The superstring standard-like models are constructed in the four dimensional free fermionic formulation [12]. The models are generated by a basis of eight bound- 
ary condition vectors for all world-sheet fermions. The first five vectors in the basis consist of the NAHE set $\left\{\mathbf{1}, S, b_{1}, b_{2}, b_{3}\right\}$ [13]. The standard-like models are constructed by adding three additional vectors to the NAHE set $[5,6]$. The observable and hidden gauge groups after application of the generalized GSO projections are $S U(3)_{C} \times U(1)_{C} \times S U(2)_{L} \times U(1)_{L} \times U(1)^{6 *}$ and $S U(5)_{H} \times S U(3)_{H} \times U(1)^{2}$, respectively. The weak hypercharge is given by $U(1)_{Y}=\frac{1}{3} U(1)_{C}+\frac{1}{2} U(1)_{L}$ and has the standard $S O(10)$ embedding. The orthogonal combination is given by $U(1)_{Z^{\prime}}=U(1)_{C}-U(1)_{L}$. The model has six right-handed and six left-handed horizontal symmetries $U(1)_{r_{j}} \times U(1)_{\ell_{j}}(j=1, \ldots, 6)$, which correspond to the right-moving and left-moving world-sheet currents respectively.

The full massless spectrum with the quantum numbers was presented in Ref. [5]. Here we list only the states that are relevant for the quark mass matrices.

(a) The $b_{1,2,3}$ sectors produce three $S O(10)$ chiral generations, $G_{\alpha}=e_{L_{\alpha}}^{c}+$ $u_{L_{\alpha}}^{c}+N_{L_{\alpha}}^{c}+d_{L_{\alpha}}^{c}+Q_{\alpha}+L_{\alpha}(\alpha=1, \cdots, 3)$

(b) The $S+b_{1}+b_{2}+\alpha+\beta$ sector gives the weak doublet $h_{45}$, the color triplet $D_{45}$ and the $S O(10)$ singlets $\Phi_{45}, \Phi_{1}^{ \pm}, \Phi_{2}^{ \pm}, \Phi_{3}^{ \pm}$and their conjugates.

(c) The Neveu-Schwarz $O$ sector gives, in addition to the graviton, dilaton, antisymmetric tensor and spin 1 gauge bosons, the scalar weak doublets $h_{1}, h_{2}, h_{3}$ and the singlets $\Phi_{12}, \Phi_{23}, \Phi_{13}$ (and their conjugates). Finally, the Neveu-Schwarz sector gives rise to three singlet states $\xi_{1,2,3}$ that are neutral under all the $\mathrm{U}(1)$ symmetries.

The sectors $b_{j}+2 \gamma+(I)(j=1, . ., 3)$ give vector-like representations that are $S U(3)_{C} \times S U(2)_{L} \times U(1)_{L} \times U(1)_{C}$ singlets and transform as $5, \overline{5}$ and $3, \overline{3}$ under the hidden $S U(5)$ and $S U(3)$ gauge groups, respectively (see Table 1). The states from the sectors $b_{j}+2 \gamma+(I)$ produce the mixing between the chiral generations [10]. In addition, they give masses to the light Higgs doublets and light quarks from non-renormalizable terms in the superpotential as we will see below.

\footnotetext{
$* U(1)_{C}=\frac{3}{2} U(1)_{B-L}$ and $U(1)_{L}=2 U(1)_{T_{3_{R}}}$.
} 
The massless spectrum also contains states from sectors with some combination of $\left\{b_{1}, b_{2}, b_{3}, \alpha, \beta\right\}$ and $\gamma+(I)$. These states are model dependent and carry either fractional electric charge or $U(1)_{Z^{\prime}}$ charge. The ones with fractional charges get large masses from non-renormalizable interactions and decouple from the spectrum. The states with $U(1)_{Z^{\prime}}$ charge are listed in Table 2 . These appear in the trilevel superpotential and mix with states from the observable sector. As a result, we will see that there are non-trivial constraints on the VEVs of these from SUSY and an aceptable Higgs doublet spectrum.

In addition to the spectrum, we have to consider the superpotential of the model. Cubic and non-renormalizable contributions to the superpotential are obtained by calculating correlators between vertex operators [9] $A_{N} \sim\left\langle V_{1}^{f} V_{2}^{f} V_{3}^{b}\right.$. $\left.\cdots V_{N}^{b}\right\rangle$ where $V_{i}^{f}\left(V_{i}^{b}\right)$ are the fermionic (bosonic) vertex operators corresponding to different fields. The non-vanishing terms are obtained by applying the rules of Ref. [9].

At the cubic level the following terms are obtained in the observable and hidden sectors [5],

$$
\begin{aligned}
W_{3} & =\left\{\left(u_{L_{1}}^{c} Q_{1} \bar{h}_{1}+N_{L_{1}}^{c} L_{1} \bar{h}_{1}+u_{L_{2}}^{c} Q_{2} \bar{h}_{2}+N_{L_{2}}^{c} L_{2} \bar{h}_{2}+u_{L_{3}}^{c} Q_{3} \bar{h}_{3}+N_{L_{3}}^{c} L_{3} \bar{h}_{3}\right)\right. \\
& +h_{1} \bar{h}_{2} \bar{\Phi}_{12}+h_{1} \bar{h}_{3} \bar{\Phi}_{13}+h_{2} \bar{h}_{3} \bar{\Phi}_{23}+\bar{h}_{1} h_{2} \Phi_{12}+\bar{h}_{1} h_{3} \Phi_{13}+\bar{h}_{2} h_{3} \Phi_{23}+\Phi_{23} \bar{\Phi}_{13} \Phi_{12} \\
& +\bar{\Phi}_{23} \Phi_{13} \bar{\Phi}_{12}+\bar{\Phi}_{12}\left(\bar{\Phi}_{1}^{+} \bar{\Phi}_{1}^{-}+\bar{\Phi}_{2}^{+} \bar{\Phi}_{2}^{-}+\bar{\Phi}_{3}^{+} \bar{\Phi}_{3}^{-}\right)+\Phi_{12}\left(\Phi_{1}^{-} \Phi_{1}^{+}+\Phi_{2}^{-} \Phi_{2}^{+}+\Phi_{3}^{-} \Phi_{3}^{+}\right) \\
& +\frac{1}{2} \xi_{3}\left(\Phi_{45} \bar{\Phi}_{45}+h_{45} \bar{h}_{45}+D_{45} \bar{D}_{45}+\Phi_{1}^{+} \bar{\Phi}_{1}^{+}+\Phi_{1}^{-} \bar{\Phi}_{1}^{-}+\Phi_{2}^{+} \bar{\Phi}_{2}^{+}+\Phi_{2}^{-} \bar{\Phi}_{2}^{-}+\Phi_{3}^{+} \bar{\Phi}_{3}^{+}\right. \\
& \left.+\Phi_{3}^{-} \bar{\Phi}_{3}^{-}\right)+h_{3} \bar{h}_{45} \Phi_{45}+\bar{h}_{3} h_{45} \bar{\Phi}_{45}+\left\{\frac { 1 } { 2 } \left[\xi_{1}\left(H_{19} H_{20}+H_{21} H_{22}+H_{23} H_{24}+H_{25} H_{26}\right)\right.\right. \\
& \left.+\xi_{2}\left(H_{13} H_{14}+H_{15} H_{16}+H_{17} H_{18}\right)\right]+\bar{\Phi}_{23} H_{24} H_{25}+\Phi_{23} H_{23} H_{26}+h_{2} H_{16} H_{17} \\
& +\bar{h}_{2} H_{15} H_{18}+e_{L_{2}}^{c} H_{8} H_{29}+\left(V_{1} H_{9}+V_{2} H_{11}\right) H_{27}+V_{6} H_{5} H_{29}+\bar{\Phi}_{45} H_{17} H_{24} \\
& \left.+D_{45} H_{18} H_{21}+h_{45} H_{16} H_{25}\right\}
\end{aligned}
$$

with a common normalization constant $\sqrt{2} g$.

There are higher order (i.e. $N>3$ ) contributions to the superpotential which 
can be calculated from the world-sheet correlators. Among other things, these give masses to the quarks, Higgs doublets $[6,14,11]$ and induce quark mixing [10] etc.

\section{SUSY}

In order to preserve SUSY at $M_{P}$, one has to satisfy a set of $\mathrm{F}$ and D constraints. The set of $\mathrm{F}$ and $\mathrm{D}$ constraints is given by the following equations:

$$
\begin{array}{ll}
D_{A} & =\sum_{k} Q_{k}^{A}\left|\chi_{k}\right|^{2}=\frac{-g^{2} e^{\phi_{D}}}{192 \pi^{2}} \operatorname{Tr}\left(Q_{A}\right) \frac{1}{2 \alpha^{\prime}} \\
D^{\prime j} & =\sum_{k} Q_{k}^{\prime j}\left|\chi_{k}\right|^{2}=0 \quad j=1 \ldots 5 \\
D^{j} & =\sum_{k} Q_{k}^{j}\left|\chi_{k}\right|^{2}=0 \quad j=C, L, 7,8 \\
W & =\frac{\partial W}{\partial \eta_{i}}=0
\end{array}
$$

where $\chi_{k}$ and $\eta_{i}$ are the fields that do and do not get VEVs respectively and $Q_{k}^{j}$ are their charges. $2 \alpha^{\prime}=g^{2} M_{P}^{2} / 8 \pi=M^{2}$ and $W$ is the superpotential. The charges $Q_{k}^{j \prime}$ correspond to linear combinations of the original local $U(1)_{r_{j}}$ which are non-anomalous whereas $Q_{A}$ corresponds to an anomalous $U(1)$ local symmetry with $\operatorname{Tr}\left(Q_{A}\right)=180[5,14]$. From the DSW mechanism, the D constraint for the anomalous $U(1)_{A}$ gets an additional term proportional to $\operatorname{Tr}\left(Q_{A}\right)$. From Eq. (6a) we see that, $S O(10)$ singlet scalars must get VEVs $\sim g^{2} M / 4 \pi \sim M / 25$ in order to preserve SUSY at $M_{P}$. The scale of the $S O(10)$ singlet VEVs is fixed by the $U(1)_{A}$ charges of the fields and the coefficient of the anomaly term. Since for a generic VEV, $\langle\Phi\rangle \sim M / 25$, we can expand the superpotential in the number of terms (inverse powers of $M$ ) where order $N$ is suppressed by an order of magnitude with respect to order $N-1$. This justifies our expansion in $N$.

The set of $\mathrm{F}$ constraints in the observable sector has been studied before [14]. One finds that SUSY requires (when either $\left\langle H_{23}\right\rangle=\left\langle H_{25}\right\rangle=0$ or $\left\langle H_{24}\right\rangle=\left\langle H_{26}\right\rangle=$ 
0 which as we will see below is the case)

$$
\left\langle\Phi_{12}\right\rangle=\left\langle\bar{\Phi}_{12}\right\rangle=\left\langle\xi_{3}\right\rangle=0
$$

even though the number of fields is larger than the number of constraints. Then, one is left with only three F constraints from the observable sector:

$$
\begin{aligned}
& \bar{\Phi}_{23} \Phi_{13}+\bar{\Phi}_{i}^{+} \bar{\Phi}_{i}^{-}=0 \\
& \bar{\Phi}_{13} \Phi_{23}+\Phi_{i}^{+} \Phi_{i}^{-}=0 \\
& \Phi_{45} \bar{\Phi}_{45}+\Phi_{i}^{+} \bar{\Phi}_{i}^{+}+\Phi_{i}^{-} \bar{\Phi}_{i}^{-}=0
\end{aligned}
$$

In the hidden sector, on the other hand, we get the following F constraints:

$$
\begin{aligned}
& H_{19} H_{20}+H_{23} H_{24}+H_{25} H_{26}=0 \\
& H_{13} H_{14}+H_{17} H_{18}=0 \\
& \frac{1}{2} \xi_{1} H_{24}+\Phi_{23} H_{26}=0 \\
& \frac{1}{2} \xi_{1} H_{23}+\bar{\Phi}_{23} H_{25}=0 \\
& \frac{1}{2} \xi_{1} H_{26}+\bar{\Phi}_{23} H_{24}=0 \\
& \frac{1}{2} \xi_{1} H_{25}+\Phi_{23} H_{23}=0 \\
& \frac{1}{2} \xi_{2} H_{13}=\frac{1}{2} \xi_{2} H_{14}=\frac{1}{2} \xi_{2} H_{17}=\frac{1}{2} \xi_{2} H_{18}=0
\end{aligned}
$$

As we will see in Section 5, the requirement of realistic (or non-zero) b, $s$, $\mu$ and $\tau$ masses means that $\xi_{1}$ and $\xi_{2}$ must get VEVs. Then, from the above $\mathrm{F}$ constraints we see that

$$
\left\langle H_{13}\right\rangle=\left\langle H_{14}\right\rangle=\left\langle H_{17}\right\rangle=\left\langle H_{18}\right\rangle=0
$$

in order to preserve SUSY at $M_{P} . H_{19}$ and $H_{20}$ are 5 and $\overline{5}$ of $S U(5)_{H}$ and obtain masses of $\left\langle\xi_{2}\right\rangle / 2 \sim M$. Therefore, they decouple from the spectrum before the hidden $S U(5)_{H}$ condenses at the scale $\Lambda_{H}$ which means that $\left\langle H_{19} H_{20}\right\rangle=0$. For the rest, $\mathrm{H}_{23}, \mathrm{H}_{24}, \mathrm{H}_{25}, \mathrm{H}_{26}$, from Eqs. (9c-f) we get the following constraints: 
a) When $\left\langle\Phi_{23}\right\rangle \neq 0$ and $\left\langle\bar{\Phi}_{23}\right\rangle \neq 0$, either $\left\langle H_{23}\right\rangle=\left\langle H_{25}\right\rangle=0$ or $\left\langle H_{24}\right\rangle=$ $\left\langle H_{26}\right\rangle=0$.

b) When one or both of $\Phi_{23}, \bar{\Phi}_{23}$ have vanishing VEVs, $\left\langle H_{23}\right\rangle=\left\langle H_{25}\right\rangle=$ $\left\langle H_{24}\right\rangle=\left\langle H_{26}\right\rangle=0$.

We stress that these SUSY constraints on the hidden sector VEVs are obtained by requiring a realistic heavy quark and lepton spectrum (i.e. $\left\langle\xi_{1,2}\right\rangle \neq 0$ ). Otherwise, Eqs. (9a-g) do not lead to useful SUSY constraints on the hidden sector VEVs.

Since $\left\langle H_{25}\right\rangle=0$ in order to have $h_{45}$ light as we will see in the next section, $\left\langle H_{23}\right\rangle=0$ in any case in this model. $\left\langle H_{24}\right\rangle$ and $\left\langle H_{26}\right\rangle$ may or may not vanish depending on the VEVs of $\Phi_{23}$ and $\bar{\Phi}_{23}$ as above.

\section{Higgs doublet masses}

The Higgs doublets in the model are $\left\{h_{1}, h_{2}, h_{3}, h_{45}\right\}$, their barred counterparts (i.e. $\bar{h}_{1}$ etc.), $H_{15}$ and $H_{16}$. The light Higgs doublets are determined by the Higgs doublet mass matrix $h_{i}\left(M_{h}\right)_{i j} \bar{h}_{j}$ where $h_{i}=\left(h_{1}, h_{2}, h_{3}, h_{45}, H_{15}\right)$ and $\bar{h}_{j}=$ $\left(\bar{h}_{1}, \bar{h}_{2}, \bar{h}_{3}, \bar{h}_{45}, H_{16}\right)$. From the cubic superpotential we get

$$
M_{h}=\left(\begin{array}{ccccc}
0 & 0 & \bar{\Phi}_{13} & 0 & 0 \\
0 & 0 & \bar{\Phi}_{23} & 0 & 0 \\
\Phi_{13} & \Phi_{23} & 0 & \Phi_{45} & 0 \\
0 & 0 & \bar{\Phi}_{45} & 0 & H_{25} \\
0 & 0 & 0 & 0 & \xi_{2}
\end{array}\right)
$$

where the SUSY constraints Eq. (7) and Eq. (10) have been taken into account. The entries in $M_{h}$ are the VEVs of the respective fields. The matrix $M_{h}$ is diagonalized by a bi-unitary transformation $\left(S M_{h} T^{\dagger}\right)_{i j}=m_{i} \delta_{i j}$. The $h$ and $\bar{h}$ eigenstates and eigenvalues are found by evaluating those of $M_{h} M_{h}^{\dagger}$ and $M_{h}^{\dagger} M_{h}$ respectively.

One sees that $H_{15}$ and $H_{16}$ will get masses (of $O(M)$ ) since $\left\langle\xi_{2}\right\rangle \neq 0$. In addition, $h_{45}$ will be heavy unless $\left\langle H_{25}\right\rangle=0 . h_{45}$ must remain massless or light 
in order to get a non-vanishing down quark mass matrix $M_{d}$. Therefore we impose $\left\langle H_{25}\right\rangle=0$ which we used in the last section. Then, generically, there are two massless doublets:one linear combination of $\left\{h_{1}, h_{2}, h_{45}\right\}$ and another linear combination of $\left\{\bar{h}_{1}, \bar{h}_{2}, \bar{h}_{45}\right\}$. Note that $h_{3}\left(\bar{h}_{3}\right)$ gets mass and decouples unless $\left\langle\Phi_{13}\right\rangle=\left\langle\Phi_{23}\right\rangle=\left\langle\Phi_{45}\right\rangle=0\left(\left\langle\bar{\Phi}_{13}\right\rangle=\left\langle\Phi_{23}\right\rangle=\left\langle\bar{\Phi}_{45}\right\rangle=0\right)$. As we will see in the next section, non-vanishing quark mixing imposes $\left\langle\Phi_{45}\right\rangle \neq 0$ so $h_{3}$ is necessarily heavy. In general, one needs to give VEVs to any or all of $\left\{\bar{\Phi}_{13}, \bar{\Phi}_{23}, \bar{\Phi}_{45}\right\}$ so that $\bar{h}_{3}$ is also heavy. The exact light doublet states depend on the specific F and D flat direction chosen for the model. For example if we take $\left\langle\bar{\Phi}_{45}\right\rangle=\left\langle\Phi_{13}\right\rangle=0$, the light doublets are $h_{45},\left(-\left\langle\bar{\Phi}_{23}\right\rangle /\left\langle\bar{\Phi}_{13}\right\rangle\right) h_{1}+h_{2}, \bar{h}_{1}$ and $\bar{h}_{2}+\left(\left\langle\Phi_{23}\right\rangle /\left\langle\Phi_{45}\right\rangle\right) \bar{h}_{45}$. Different flat directions with two more vanishing VEVs give different linear combinations which remain light. Thus, when two more scalar VEVs (in addition to those that vanish due to SUSY constraints) are zero, there are two combinations of $h_{i}$ and $\bar{h}_{i}$ each which remain light. One doublet of each kind must get mass at an intermediate scale lest there is the danger of large contributions to flavor changing neutral currents from Higgs exchanges. Since this question is not directly related to the problem we examine, we simply assume that this is the case and not expand on this point further.

Higgs doublets also get masses from higher order non-renormalizable terms. Since only $\bar{h}_{1}$ or $\bar{h}_{2}$ and $h_{45}$ are relevant for quark mass matrices we list only the terms involving them. There are terms which contain hidden sector states with $U(1)_{Z^{\prime}}$ charge such as

$$
h_{2} \bar{h}_{45} \Phi_{45} H_{23} H_{26} \quad \bar{h}_{2} h_{45} \bar{\Phi}_{45} H_{24} H_{25}
$$

Similar terms for $h_{1}$ and $\bar{h}_{1}$ do not exist because of $U(1)_{r_{1}}$ conservation. The potentially dangerous terms $(12 \mathrm{a}, \mathrm{b})$ vanish due to SUSY constraints in the hidden sector. In addition, there are terms which contain states from the sectors $b_{j}+2 \gamma+$ (I) such as

$$
h_{1} \bar{h}_{2} \Phi_{45} \bar{\Phi}_{1}^{+} V_{1} \bar{V}_{1} \quad h_{2} \bar{h}_{1} \Phi_{45} \Phi_{2}^{-} V_{2} \bar{V}_{2}
$$


Also there are terms which induce a mixing i.e. $\mu$ terms such as

$$
\begin{array}{ll}
\bar{h}_{1} h_{45} \bar{\Phi}_{3}^{-} T_{3} \bar{T}_{3} & \bar{h}_{2} h_{45} \Phi_{3}^{+} T_{3} \bar{T}_{3} \\
\bar{h}_{1} h_{45} \Phi_{3}^{-} V_{3} \bar{V}_{3} & \bar{h}_{2} h_{45} \bar{\Phi}_{3}^{+} V_{3} \bar{V}_{3}
\end{array}
$$

In order to get enough quark mixing, one needs $\left\langle V_{j}\right\rangle \sim 10^{16} \mathrm{GeV}$. This means that $\left\langle V_{j}\right\rangle$ does not arise from condensation of $S U(3)_{H}$ (since $\Lambda_{H} \sim 10^{10} \mathrm{GeV}$ for this model) but from the $\mathrm{D}$ constraints [10]. The scale of scalar VEVs which arise from the D constraints is $\sim M / 25 \sim 10^{16} \mathrm{GeV}$ as fixed by the right hand side of Eq. (6a). From Eqs. (13a,b) and higher order terms of the same kind we see that unless we choose to give VEVs only to one $V_{j}$ from each sector $j$, we get very large masses for the doublets which couple to up-like quarks. In that case, they decouple from the low-energy spectrum and up-like quarks cannot get masses. This is phenomenologically unacceptable, so we give VEVs to only one $V_{j}$ from each sector $b_{j}+2 \gamma+(I)$. Thus, the dangerous terms in Eqs. (13a,b) and $(14 \mathrm{c}, \mathrm{d})$ vanish.

From Eq. (14a,b) we see that the Higgs mixing terms due to $\left\langle T_{3} \bar{T}_{3}\right\rangle$ give an unacceptably large $\mu \sim 10^{8} \mathrm{GeV}$ for $\Lambda_{H} \sim 10^{14} \mathrm{GeV}$. As a result, when $\bar{h}_{1}\left(\bar{h}_{2}\right)$ is light, we have to take $\left\langle\bar{\Phi}_{3}^{-}\right\rangle=0\left(\left\langle\Phi_{3}^{+}\right\rangle=0\right)$. Then, Higgs mixing will arise from higher order terms which are smaller and acceptable.

\section{Light Quark Masses}

At the cubic order of the superpotential, there is only a mass term for the heaviest up quark i.e. the top when only $\bar{h}_{1}$ or $\bar{h}_{2}$ remains light. The light Higgs doublet with $Q_{L}=1$ determines the heaviest generation. At the quartic order there are no potential quark mass terms. At the quintic order the following mass terms are obtained [11],

$$
\begin{aligned}
& d_{1} Q_{1} h_{45} \Phi_{1}^{+} \xi_{2} \quad d_{2} Q_{2} h_{45} \bar{\Phi}_{2}^{-} \xi_{1} \\
& u_{1} Q_{1}\left(\bar{h}_{45} \Phi_{45} \bar{\Phi}_{13}+\bar{h}_{2} \Phi_{i}^{+} \Phi_{i}^{-}\right)
\end{aligned}
$$




$$
\begin{aligned}
& u_{2} Q_{2}\left(\bar{h}_{45} \Phi_{45} \bar{\Phi}_{23}+\bar{h}_{1} \bar{\Phi}_{i}^{+} \bar{\Phi}_{i}^{-}\right) \\
& \left(u_{1} Q_{1} h_{1}+u_{2} Q_{2} h_{2}\right) \frac{\partial W}{\partial \xi_{3}} .
\end{aligned}
$$

The mass term from the cubic superpotential and the terms above give the mass hierarchy between the two heavy quark generations. The heaviest up-like quark mass term appears at $N=3$ whereas the mass terms for the other quarks (except for the light ones) appear at $N=5$. The latter are suppressed by a factor of $\Phi^{2} / M^{2} \sim(1 / 25)^{2}$ relative to the former and as a result one can easily obtain the two or three orders of magnitude suppression required. The analysis of the non-renormalizable terms up to order $N=8$ shows that quark mixing terms are obtained for all generations [10]. The full list of terms that give mixing between the quarks have been given in Ref. (10) and will not be repeated here. In addition there are lepton mass terms[14]

$$
e_{1} L_{1} h_{45} \Phi_{1}^{-} \xi_{2} \quad e_{2} L_{2} h_{45} \bar{\Phi}_{2}^{+} \xi_{1}
$$

There are two possible scenarios with $\bar{h}_{1}$ or $\bar{h}_{2}$ as the light doublet that couples to the up-like quarks. (An additional possibility is that a linear combination of the two remains light. That case is easy to examine once these two are understood.) In both cases the down-like quarks couple to $h_{45}$ because of $Q_{\ell_{j}}$ quantum numbers. In the first case, with $\bar{h}_{1}$ and $h_{45}$ as the light doublets, we get with a suitable set of $S O(10)$ singlets with VEVs[10] (and the SUSY constraints from Eq. (7))

$$
M_{u} \sim\left(\begin{array}{ccc}
\epsilon_{1} & \frac{V_{3} \bar{V}_{2} \Phi_{45} \bar{\Phi}_{3}^{+}}{M^{4}} & 0 \\
\frac{V_{3} \bar{V}_{2} \Phi_{45} \bar{\Phi}_{2}^{+}}{M^{4}} & \frac{\bar{\Phi}_{i}^{-} \bar{\Phi}_{i}^{+}}{M^{2}} & \frac{V_{1} \bar{V}_{2} \Phi_{45} \bar{\Phi}_{2}^{+}}{M^{4}} \\
0 & \frac{V_{1} \bar{V}_{2} \Phi_{45} \bar{\Phi}_{1}^{+}}{M^{4}} & 1
\end{array}\right)\left\langle\bar{h}_{1}\right\rangle
$$

and

$$
M_{d} \sim\left(\begin{array}{ccc}
\epsilon_{2} & \frac{V_{3} \bar{V}_{2} \Phi_{45}}{M^{3}} & 0 \\
\frac{V_{3} \bar{V}_{2} \Phi_{45} \xi_{1}}{M^{4}} & \frac{\bar{\Phi}_{2}^{-} \xi_{1}}{M^{2}} & \frac{V_{1} \bar{V}_{2} \Phi_{45} \xi_{i}}{M^{4}} \\
0 & \frac{V_{1} \bar{V}_{2} \Phi_{45} \xi_{i}}{M^{4}} & \frac{\Phi_{1}^{+} \xi_{2}}{M^{2}}
\end{array}\right)\left\langle h_{45}\right\rangle
$$

where $\epsilon_{1,2}$ are small numbers to be determined below. In the second case, where 
the light doublets are $\bar{h}_{2}$ and $h_{45}$, we get with the same set of VEVs[10]

$$
M_{u} \sim\left(\begin{array}{ccc}
\epsilon_{1} & \frac{V_{3} \bar{V}_{1} \Phi_{45} \Phi_{3}^{-}}{M^{4}} & 0 \\
\frac{V_{3} \bar{V}_{1} \Phi_{45} \Phi_{1}^{-}}{M^{4}} & \frac{\Phi_{i}^{-} \Phi_{i}^{+}}{M^{2}} & \frac{V_{2} \bar{V}_{1} \Phi_{45} \Phi_{1}^{-}}{M^{4}} \\
0 & \frac{V_{2} \bar{V}_{1} \Phi_{45} \bar{\Phi}_{2}^{+}}{M^{4}} & 1
\end{array}\right)\left\langle\bar{h}_{2}\right\rangle
$$

and

$$
M_{d} \sim\left(\begin{array}{ccc}
\epsilon_{2} & \frac{V_{3} \bar{V}_{1} \Phi_{45}}{M^{3}} & 0 \\
\frac{V_{3} \bar{V}_{1} \Phi_{45} \xi_{2}}{M^{4}} & \frac{\bar{\Phi}_{1}^{+} \xi_{2}}{M^{2}} & \frac{V_{2} \bar{V}_{1} \Phi_{45} \xi_{i}}{M^{4}} \\
0 & \frac{V_{2} \bar{V}_{1} \Phi_{45} \xi_{i}}{M^{4}} & \frac{\bar{\Phi}_{2}^{-} \xi_{1}}{M^{2}}
\end{array}\right)\left\langle h_{45}\right\rangle
$$

As explained in Ref. (10) the texture of $M_{u}$ and $M_{d}$ is a result of the choice of $V_{j}, \bar{V}_{j}$ s which get VEVs. (We remind that only one $V_{j}, \bar{V}_{j}$ from each sector $b_{j}+2 \gamma$ can get a VEV due to constraints from Higgs doublet masses.) Once the VEVs are chosen, there is an effective discrete symmetry which protects the zeros in $M_{u}$ and $M_{d}$ except for light quark masses (i.e. $\epsilon_{1,2}$ ). The up and down quark mass matrices are diagonalized by bi-unitary transformations $U_{L} M_{u} U_{R}^{\dagger}=D_{u} \equiv \operatorname{diag}\left(m_{u}, m_{c}, m_{t}\right)$ and $D_{L} M_{d} D_{R}^{\dagger}=D_{d} \equiv \operatorname{diag}\left(m_{d}, m_{s}, m_{b}\right)$ with the CKM mixing matrix given by $V=U_{L} D_{L}^{\dagger}$. Since we are only interested in order of magnitude results, we will loosely take $\tan \beta=\left\langle\bar{h}_{1,2}\right\rangle /\left\langle h_{45}\right\rangle \sim 1$ in both cases.

From Eqs. (15a-e) and the mass matrices we see that in both cases the light generation is fixed to be the one with index 3 since it is by far the lightest. The heaviest generation is the one with index 1 (2) for the first (second) case since the light Higgs doublet which couples to up-like quarks in the cubic superpotential is $\bar{h}_{1}\left(\bar{h}_{2}\right)$. If we consider only the observable sector of the spectrum, we find that there are potential $Q_{3} u_{3}$ and $Q_{3} d_{3}$ terms such as

$$
Q_{3} u_{3} \bar{h}_{1} \Phi_{13} \xi_{3} \quad Q_{3} d_{3} h_{45} \bar{\Phi}_{13} \Phi_{3}^{+} \xi_{3}
$$

These terms potentially induce very large masses to up and down quarks. (For generic VEVs, they give $m_{u} \sim G e V$ and $m_{d} \sim 100 \mathrm{MeV}$.) Fortunately, they vanish due to the SUSY constraints in the observable sector as given by Eq. (7). 
The SUSY constraints gaurantee that all potential $Q_{3} u_{3}$ or $Q_{3} d_{3}$ terms, arising only from the observable states, vanish to all orders in $N$ as follows [11]. $Q_{3}, u_{3}$ and $d_{3}$ have $Q_{\ell_{3}}=1 / 2$ and the only $S O(10)$ singlets with $Q_{\ell_{3}}$ charges in the observable sector are $\Phi_{12}, \bar{\Phi}_{12}$ and $\xi_{3}$ (since $h_{3}, \bar{h}_{3}$ get very large masses and decouple at low energies). If these cannot get VEVs, then there cannot be non-vanishing $Q_{3} u_{3}$ or $Q_{3} d_{3}$ terms to any order $N$ due to conservation of $Q_{\ell_{3}}$. Actually the fact that these terms vanish is welcome since if they did not they would result in up and down quark masses which are too large.

Of course, there are other contributions once hidden sector states are taken into account. For example, at $N=5$ and $N=6$, there are terms containing hidden sector states with $U(1)_{Z^{\prime}}$ charge such as

$$
Q_{3} u_{3} \bar{h}_{45} H_{17} H_{24} \quad Q_{3} d_{3} h_{45} \Phi_{3}^{+} H_{24} H_{25}
$$

which potentially induce very large up and down masses (similar to those from Eq. (21a,b) in magnitude). These, too, vanish due to the SUSY constraints in the hidden sector (with the additional requirement for a realistic heavy quark spectrum). In both cases, when $H_{23}, H_{25}$ or $H_{24}, H_{26}$ have vanishing VEVs, the terms in Eq. (22a,b) and all similar higher order terms containing $H_{i}$ vanish. Finally, we have terms which contain states from the hidden sectors $b_{j}+2 \gamma+(I)$. At $N=8$ we get terms with $T_{j} \bar{T}_{j}$ and $V_{j} \bar{V}_{j}$

$$
\begin{gathered}
Q_{3} u_{3} \bar{h}_{1} \Phi_{3}^{+} \Phi_{45} \Phi_{13} T_{3} \bar{T}_{3} \\
Q_{3} u_{3} \bar{h}_{2} \Phi_{3}^{-} \Phi_{45} \Phi_{13} T_{3} \bar{T}_{3} \\
Q_{3} d_{3} h_{45} \bar{\Phi}_{3}^{+} \Phi_{3}^{-} \Phi_{45} T_{3} \bar{T}_{3} \\
Q_{3} u_{3} \bar{h}_{1} \Phi_{3}^{-} \Phi_{45} \Phi_{13} V_{3} \bar{V}_{3} \\
Q_{3} u_{3} \bar{h}_{2} \Phi_{3}^{+} \Phi_{45} \Phi_{13} V_{3} \bar{V}_{3} \\
Q_{3} d_{3} h_{45} \bar{\Phi}_{3}^{-} \Phi_{3}^{-} \Phi_{45} V_{3} \bar{V}_{3}
\end{gathered}
$$

The terms containing $V_{3} \bar{V}_{3}$ can potentially induce $m_{u}$ and $m_{d}$ of $O(M e V)$. These vanish since only one $V_{j}$ or $\bar{V}_{j}$ from every sector can get a VEV as we saw before. 
When $\bar{h}_{2}$ remains light, the term in Eq. (23a) vanishes due to the $\mu$ constraint, Eq. (9b), which gives $\left\langle\Phi_{3}^{+}\right\rangle=0$. The other two terms are non-vanishing in general and are $\sim O\left(10^{-6} \mathrm{MeV}\right)$ for $\Lambda_{H} \sim 10^{14} \mathrm{GeV}$. We see that light quark masses get contributions either from the terms $(23 \mathrm{a}-\mathrm{c})$ or from the off-diagonal terms in $M_{u}$ and $M_{d}$ through diagonalization.

We stress that the potentially large up and down mass terms in Eqs. (21) and (22) vanish because of the SUSY constraints in the observable and hidden sectors. The set of vanishing VEVs due to SUSY results in an effective $Z_{4}$ symmetry under which $Q_{3}, u_{3}, d_{3}$ have charge $\alpha$ and $T_{j}, \bar{T}_{j}, V_{j}, \bar{V}_{j}$ have charge $\alpha^{3}\left(\alpha^{4}=1\right)$. This discrete symmetry eliminates the terms in Eqs. (21) and (22). Then, the only potential up and down mass terms come either from Eqs. (23a-f) or from the mixing terms in $M_{u}$ and $M_{d}$.

We analyze the two cases seperately with the requirement $\sin \theta_{c} \sim 0.2$. In the first case, when the light doublets are $\bar{h}_{1}$ and $h_{45}, M_{u}$ and $M_{d}$ are given by Eqs. (17) and (18) respectively. Quark mixing arises mainly from $M_{d}$ and as a result we have

$$
\sin \theta_{c} \sim \frac{\left(M_{d}\right)_{12}}{\left(M_{d}\right)_{22}} \quad \text { and } \quad m_{d} \sim \frac{\left(M_{d}\right)_{12}\left(M_{d}\right)_{21}}{\left(M_{d}\right)_{22}}
$$

Therefore, $m_{d} \sim 0.2 \times\left(M_{d}\right)_{21}$. Since $\left(M_{d}\right)_{21} \sim\left(M_{d}\right)_{12}\left\langle\xi_{1}\right\rangle$ and $\left(M_{d}\right)_{22}=m_{s} \sim$ $150 \mathrm{MeV}$, we find that $m_{d} \sim 4 \times 10^{-5}\left\langle\xi_{1}\right\rangle\left\langle h_{45}\right\rangle / M$. $\xi_{1}$ does not appear in F or D constraints and therefore we can choose $\left\langle\xi_{1}\right\rangle \sim M$ which gives $m_{d} \sim 4 \mathrm{MeV}$, of the correct order of magnitude. (From $m_{\mu} \sim 100 \mathrm{MeV}$ we find that $\left\langle\xi_{1}\right\rangle \sim M$ requires $\left\langle\bar{\Phi}_{2}^{-}\right\rangle \sim 10^{-3} M$ which is certainly possible but an order of magnitude smaller than its natural value, $M / 25$.) One might think that the appearance of a large VEV, i.e. $\left\langle\xi_{1}\right\rangle \sim M$, destroys the perturbative expansion in $N$. One can simply form terms at $N+1$ from terms at $N$ by adding $\xi_{1}$. This is not the case because string (or world-sheet) selection rules require that, whenever one adds $\xi_{1}$ to a non-vanishing string of fields, another field (with $\mathrm{VEV} \sim M / 25$ ) accompany it. Then, one can form terms only at order $N+2$ (by adding $\xi_{1}$ to an order $N$ term) and these are suppressed relative to order $N$ terms by at least an order of magnitude. Note that 
the contribution of the terms in Eq. (23a-c) is extremely small $\left(\sim 10^{-6} \mathrm{MeV}\right.$ !) compared to the above.

The situation for $m_{u}$ is different. We find that $m_{u} \sim\left(M_{u}\right)_{12}\left(M_{u}\right)_{21} /\left(M_{u}\right)_{22}$ where

$$
\left(M_{u}\right)_{12} \sim\left(M_{d}\right)_{12} \frac{\langle\Phi\rangle}{M} \sim 2 \times 10^{-4}\left\langle h_{45}\right\rangle \frac{\langle\Phi\rangle}{M}
$$

On the other hand, since $\left\langle\xi_{1}\right\rangle \sim M$, from the $m_{\mu}$ term, Eq.(16b), we obtain $\left\langle\bar{\Phi}_{2}^{+}\right\rangle \sim 10^{-3} M$. Using $\left(M_{u}\right)_{22}=m_{c} \sim 1.5 \mathrm{GeV}$ we get $m_{u} \sim 10^{-5} \mathrm{MeV}$ which is six orders of magnitude smaller than the current up mass and too large to solve the strong $\mathrm{CP}$ problem naturally. In order to solve the strong $\mathrm{CP}$ problem one needs $[2]$

$$
\theta_{t o t} \frac{z}{1+z}<10^{-9}
$$

where $\theta_{\text {tot }}=\theta_{Q C D}+\theta_{\text {quarks }}$ and $z=m_{u} / m_{d}$. For the current quark masses $m_{u} \sim 5 \mathrm{MeV}$ and $m_{d} \sim 9 \mathrm{MeV}$ one needs $\theta_{t o t}<10^{-9}$ which is the strong CP problem. It has been shown that there are no axions which can solve the strong CP problem in standard-like superstring models [15]. An alternative solution is $\theta_{\text {tot }}$ of $O(1)$ and $z<10^{-9}$ which requires a very small or vanishing up quark mass. We remind that such a small $m_{u}$ is compatible with current algebra results [3]. Note that since quark mixing comes from $M_{d}$, there is no constraint (lower bound) on elements of $M_{u}$ from $\sin \theta_{c}$. It is the constraint $\sin \theta \sim 0.2$ and the existence of $\left\langle\xi_{1}\right\rangle$ which can get a large $(\sim M) \mathrm{VEV}$ in $\left(M_{d}\right)_{21}$ that allows for an acceptable $m_{d}$

Another possibility is the case with $\left\langle\bar{\Phi}_{3}^{+}\right\rangle=0 .\left\langle\bar{\Phi}_{2}^{+}\right\rangle\left(\right.$or $\left.\left(M_{u}\right)_{21}\right)$ cannot vanish because it appears in the $m_{\mu}$ term given by Eq. $(16 \mathrm{~b}) \cdot\left\langle\bar{\Phi}_{3}^{+}\right\rangle\left(\right.$or $\left.\left(M_{u}\right)_{21}\right)$ on the other hand, can vanish since it does not appear in any term that must be nonzero. The only constraints on $\left\langle\bar{\Phi}_{3}^{+}\right\rangle$are the $\mathrm{F}$ and $\mathrm{D}$ constraints and these can be satisfied whether $\left\langle\bar{\Phi}_{3}^{+}\right\rangle$vanishes or not. When $\left\langle\bar{\Phi}_{3}^{+}\right\rangle=0$ the leading contribution to $m_{u}$ comes from the hidden sector condensate term in Eq. (23a). This gives $m_{u} \sim 10^{-6} \mathrm{MeV}$ which is an order of magnitude smaller than before but still not 
small enough to solve the strong CP problem naturally. If, on the other hand, we choose a flat direction with $\left\langle\Phi_{3}^{ \pm}\right\rangle=\left\langle\bar{\Phi}_{3}^{ \pm}\right\rangle=0$, then $m_{u}=0$ to up to $N=8$. In this case there are no contributions to up mass either from $Q_{3} u_{3}$ terms or from mixing in $M_{u}$ up to $N=8$. Does this mean that $m_{u}=0$ to all orders in $N$ for this flat direction? The answer is no since there are higher order contributions to up mass from $Q_{3} u_{3}$ terms (from $T_{1} \bar{T}_{1}$ and $T_{2} \bar{T}_{2}$ condensates) and mixing in $M_{u}$ from $N>8$. In fact there are $N=9$ terms which cannot vanish due to other phenomenological constraints such as heavy quark and lepton masses etc. The non-vanishing $N=9$ terms give $m_{u} \sim 10^{-7} \mathrm{MeV}$ which can be taken as a lower bound in this model. Quite generally, even if we choose a flat direction for which $m_{u}$ vanishes up to some order in $N$, there will be non-vanishing contributions from higher orders. There are no flat directions for which $m_{u}$ vanishes to all $N$ if we demand realistic heavy quark and lepton masses.

In the second case, where the light doublets are $\bar{h}_{2}$ and $h_{45}, M_{u}$ and $M_{d}$ are given by Eqs. (19)and (20) respectively. Again

$$
\sin \theta_{c} \sim \frac{\left(M_{d}\right)_{12}}{\left(M_{d}\right)_{22}} \quad \text { and } \quad m_{d} \sim \frac{\left(M_{d}\right)_{21}\left(M_{d}\right)_{12}}{\left(M_{d}\right)_{22}}
$$

but now the elements of $M_{u}$ and $M_{d}$ are different. (Now generation with index 2 is the heaviest.) Requiring $\sin \theta_{c} \sim 0.2$ and taking $\left\langle\xi_{2}\right\rangle \sim M$, we find once again $m_{d} \sim 4 \times 10^{-5}\left\langle\xi_{1}\right\rangle\left\langle h_{45}\right\rangle / M$ which gives $m_{d} \sim 4 \mathrm{MeV}$. Now, as in the first case, $m_{u}$ vanishes if either $\left(M_{u}\right)_{12}$ or $\left(M_{u}\right)_{21}$ vanishes. $\left(M_{u}\right)_{21}$ is non-zero because $\left\langle\Phi_{1}^{-}\right\rangle$appears in $m_{\mu} .\left(M_{u}\right)_{12}$, on the other hand, can vanish since $\left\langle\Phi_{3}^{-}\right\rangle$does not have to get a VEV. The situation is similar to the first case and using the same arguments we find that $m_{u} \sim 10^{-5} \mathrm{MeV}$ if $\left\langle\Phi_{3}^{-}\right\rangle \neq 0$. When $\left\langle\Phi_{3}^{-}\right\rangle=0, m_{u}$ arises mainly from the $Q_{3} u_{3}$ terms containing the hidden sector condensates which give $m_{u} \sim 10^{-6} \mathrm{MeV}$ as before. The previous comments on solving the strong $\mathrm{CP}$ problem and a vanishing $m_{u}$ to all orders for some flat direction for the first case hold for this case too.

\section{Discussion and Conclusions}


We examined the light quark masses in a standard-like superstring model in this paper. Our results are as follows. An acceptable $m_{d}$ (i.e. $\sim 5 \mathrm{MeV}$ ) can be obtained for a family of flat directions with $\left\langle\xi_{1}\right\rangle \sim M$ (or $\left\langle\xi_{2}\right\rangle \sim M$ ). $m_{d}$ arises from the off-diagonal terms in the down quark mass matrix. This is a result of the fact that quark mixing arises mainly from $M_{d}$ and $\sin \theta_{c} \sim 0.2$. Then, $\left(M_{d}\right)_{12}$ is more or less fixed since $\sin \theta_{c} \sim\left(M_{d}\right)_{12} / m_{s}$. In addition $\left(M_{d}\right)_{21}=\left(M_{d}\right)_{12}\left\langle\xi_{2}\right\rangle$ where $\left\langle\xi_{2}\right\rangle$ is free since it does not appear in $\mathrm{F}$ and $\mathrm{D}$ constraints. Thus, we can take $\left\langle\xi_{2}\right\rangle \sim M$ and obtain an $M e V$ scale $m_{d}$.

The situation for $m_{u}$ is different. There are no constraints from quark mixing on $\left(M_{u}\right)_{12}$ and $\left(M_{u}\right)_{21}$ so they are in general much smaller. Moreover, $\left\langle\xi_{2}\right\rangle$ is replaced with $\left\langle\bar{\Phi}_{2}^{+}\right\rangle$and $\left\langle\bar{\Phi}_{3}^{+}\right\rangle$(or with $\Phi_{1}^{-}$and $\Phi_{3}^{-}$in the second case) which are about $M / 10^{3}$ and $M / 10$ respectively. Consequently, from the off-diagonal terms in $M_{u}$, we find that, in either case, $m_{u} \sim 10^{-5} \mathrm{MeV}$ which is six orders of magnitude smaller than the current up mass. We stress that, in standard-like superstring models, $m_{u}$ cannot be as large as its value from current algebra. Still, such a small up mass is not in conflict with current algebra results. $m_{u}$ though very small, is not small enough to solve the strong CP problem naturally. In the first (second) case $m_{u}$ can be an order of magnitude smaller if $\left\langle\bar{\Phi}_{3}^{+}\right\rangle=0\left(\left\langle\Phi_{3}^{-}\right\rangle=0\right)$. In that case, $m_{u}$ arises not from the off-diagonal terms in $M_{u}$ but from $N=8$ terms with hidden sector condensates and $m_{u}<10^{-6} \mathrm{MeV}$. We also find that for a $\mathrm{F}$ and $\mathrm{D}$ flat direction with $\left\langle\Phi_{3}^{ \pm}\right\rangle=\left\langle\bar{\Phi}_{3}^{ \pm}\right\rangle=0, m_{u}=0$ up to $N=8$ but not for all $N$ since there are always some non-zero $N=9$ terms which contribute to $m_{u}$. As a result, $m_{u}$ cannot vanish in this model. The $N=9$ terms give $m_{u} \sim 10^{-7} \mathrm{MeV}$ which still is not small enough to solve the strong CP problem naturally.

Why are the light quark masses so small compared to other quark masses or the weak scale? We see that SUSY preservation in the observable and hidden sectors, with the requirement of realistic heavy quark and lepton masses, plays a very important role in this respect. In general, there are potentially large mass terms for $u$ and $d$ from states in the observable sector. These vanish to all orders due to the SUSY constraints in the observable sector. There are additional terms 
coming from the hidden sector states with $U(1)_{Z^{\prime}}$ charge. These, too, vanish due to SUSY constraints in the hidden sector (using the requirement of a realistic heavy quark and lepton spectrum). As a result, the only non-vanishing $Q_{3} u_{3}$ and $Q_{3} d_{3}$ terms arise from VEVs of hidden sector condensates $\left\langle T_{j} \bar{T}_{j}\right\rangle$ which break SUSY dynamically in the hidden sector or from terms containing $\left\langle V_{j} \bar{V}_{j}\right\rangle$. The former are extremely small $\left(\sim 10^{-6} \mathrm{MeV}\right)$ since they are suppressed by $\left(\Lambda_{H}^{2} / M^{2}\right) \sim 10^{-8}$ with respect to the others. The latter which can potentially give $\mathrm{MeV}$ scale up and down masses vanish due to the constraints from an acceptable Higgs doublet spectrum.

There are also contributions to $m_{u}$ and $m_{d}$ from the off-diagonal terms in up and down quark mass matrices (through diagonalization). These off-diagonal elements or mixing terms arise from VEVs of hidden sector states $\left(V_{i} \bar{V}_{j}\right.$ etc.). In any case, the mixing terms are small compared to the diagonal (or mass) terms i.e. $\left(M_{u, d}\right)_{i j}<<\left(M_{u, d}\right)_{i i}(1<i, j<3)$ except for the 33 terms, that is except for the light quark mass terms. As a result, the contribution of the mixing terms to the light quark masses is much smaller than other quark masses.

Why is $m_{u}$ so much smaller than $m_{d}$ in this model? One reason is the different contributions coming from the mixing terms in the mass matrices as explained above. The other is the elimination of the terms containing $\left\langle V_{j} \bar{V}_{j}\right\rangle$ due to the constraints from an acceptable Higgs doublet spectrum. These terms are the only ones which give an $\mathrm{MeV}$ scale contribution to $m_{u}$ in this model.

Our results can also be explained by using symmetry arguments. SUSY constraints in the observable and hidden sectors force a number of VEVs to vanish. This, in turn, results in an effective $Z_{4}$ symmetry (with parameter $\alpha$, where $\left.\alpha^{4}=1\right)$. Under this $Z_{4}, Q_{3}, u_{3}, d_{3}$ have charge $\alpha$ and $T_{j}, \bar{T}_{j}, V_{j}, \bar{V}_{j}$ have charge $\alpha^{3}$. Then, the only potential up and down mass terms come from terms which contain $\left\langle T_{j} \bar{T}_{j}\right\rangle$ and $\left\langle V_{j} \bar{V}_{j}\right\rangle$ or from mixing terms as we saw. Once different $T_{j}$ and $V_{j}$ get VEVs, the $Z_{4}$ symmetry is broken and up and down quarks obtain masses.

We conclude that, in the standard-like superstring model considered, $m_{u}$ can- 
not be as large as a few $M e V s$. In fact it is at most $10^{-5} \mathrm{MeV}$. For a family of flat directions or vacua $m_{u}$ can be as small as $10^{-7} \mathrm{MeV}$ but not much smaller. This range of up masses i.e. $10^{-7} \mathrm{MeV}<m_{u}<10^{-5} \mathrm{MeV}$ cannot solve the strong CP problem naturally but is compatible with current algebra results. An acceptable $m_{d}$ at the $\mathrm{MeV}$ scale can easily be obtained. We find that the smallness of the light quark masses relative to the other quark masses is connected to supersymmetry preservation in the observable and hidden sectors and the requirement for a realistic haevy quark and lepton spectrum.

\section{Acknowledgments}

This work is supported by a Feinberg Fellowship and the Department of Physics. I thank Alon Faraggi for many useful discussions on this subject. I also thank Yosi Nir for correcting a few points. 


\section{REFERENCES}

1. G. 't Hooft, in Recent Developements in Gauge Theories, ed. G. 't Hooft et. al. (Plenum, New York, 1980).

2. J.E. Kim, Phys. Rep. 150 (1987) 1; H.Y. Cheng, Phys. Rep. 158 (1988) 1.

3. H. Georgi and I. N. McArthur, Harvard University Report HUTP-81/A001 (1981), unpublished: D. Kaplan and A. Manohar, Phys. Rev. Lett. 56 (1986) 2004.

4. M. Green, J. Schwarz and E. Witten, Superstring Theory, 2 vols., Cambridge University Press, 1987.

5. A.E. Faraggi, Phys. Lett. B 278 (1992) 131.

6. A.E. Faraggi, Phys. Lett. B 274 (1992) 47; Nucl. Phys. B 387 (1992) 289.

7. M. Dine, N. Seiberg and E. Witten, Nucl. Phys. B 289 (1987) 585.

8. J.J. Atick, L.J. Dixon and A. Sen, Nucl. Phys. B 292 (1987) 109; S. Cecotti, S. Ferrara and M. Villasante, Int. J. Mod. Phys. A 2 (1987) 1839.

9. S. Kalara, J. Lopez and D.V. Nanopoulos, Phys. Lett. B 245 (1991) 421; Nucl. Phys. B 353 (1991) 650.

10. A. E. Faraggi and E. Halyo, Phys. Lett. B 307 (1993) 305; WIS-93/34/MAR$\mathrm{PH}$.

11. A.E. Faraggi, WIS-92/81/OCT-PH, to appear in Nucl. Phys. B.

12. I. Antoniadis, C. Bachas, and C. Kounnas, Nucl. Phys. B 289 (1987) 87; I. Antoniadis and C. Bachas, Nucl. Phys. B 298 (1988) 586; 5H. Kawai, D.C. Lewellen, and S.H.-H. Tye, Phys. Rev. Lett. 57 (1986) 1832; Phys. Rev. D 34 (1986) 3794; Nucl. Phys. B 288 (1987) 1; R. Bluhm, L. Dolan, and P. Goddard, Nucl. Phys. B 309 (1988) 330.

13. I. Antoniadis, J. Ellis, J. Hagelin, and D. V. Nanopoulos, Phys. Lett. B 231 (1989) 65. 
14. A.E. Faraggi, Nucl. Phys. B 403 (1993) 101.

15. E. Halyo, WIS-93/66/JUL-PH, to appear in Physics Letters B. 